\title{
A Novel Iterative Discrete Estimation Algorithm for Low-Complexity Signal Detection in Uplink Massive MIMO Systems
}

\author{
Hui Feng ${ }^{1}$, Xiaoqing Zhao ${ }^{2}$, Zhengquan $\mathrm{Li}^{2, *}$ and Song Xing ${ }^{3}$ \\ 1 Department of Electronic Science and Technology, Tongji University, Shanghai 201804, China \\ 2 Jiangsu Provincial Engineering Laboratory of Pattern Recognition and Computational Intelligence, \\ Jiangnan University, Wuxi 214122, China \\ 3 Department of Information Systems, California State University, Los Angeles, CA 90032, USA \\ * Correspondence: lzq722@jiangnan.edu.cn; Tel.: +86-0510-8591-0633
}

Received: 1 August 2019; Accepted: 29 August 2019; Published: 2 September 2019

check for updates

\begin{abstract}
In this paper, a novel iterative discrete estimation (IDE) algorithm, which is called the modified IDE (MIDE), is proposed to reduce the computational complexity in MIMO detection in uplink massive MIMO systems. MIDE is a revision of the alternating direction method of multipliers (ADMM)-based algorithm, in which a self-updating method is designed with the damping factor estimated and updated at each iteration based on the Euclidean distance between the iterative solutions of the IDE-based algorithm in order to accelerate the algorithm's convergence. Compared to the existing ADMM-based detection algorithm, the overall computational complexity of the proposed MIDE algorithm is reduced from $O\left(N_{t}^{3}\right)+O\left(N_{r} N_{t}^{2}\right)$ to $O\left(N_{t}^{2}\right)+O\left(N_{r} N_{t}\right)$ in terms of the number of complex-valued multiplications, where $\mathrm{Ntand} \mathrm{Nr}$ are the number of users and the number of receiving antennas at the base station (BS), respectively. Simulation results show that the proposed MIDE algorithm performs better in terms of the bit error rate (BER) than some recently-proposed approximation algorithms in MIMO detection of uplink massive MIMO systems.
\end{abstract}

Keywords: massive MIMO systems; MIDE algorithm; low computational complexity; BER

\section{Introduction}

With the development of the mobile Internet and the Internet of Things, much high data rate communication is required in the new generation of cellular networks like 5G [1]. By equipping hundreds of antennas at the base station (BS) serving tens of users, the massive multiple-input multiple-output (MIMO) is deemed one key technology for meeting the 5G requirements due to its improvements in data throughput, link reliability, higher spectral efficiency, and better communication quality compared with the traditional MIMO usage [2-4].

However, when applying the massive MIMO, a major computational challenge is the data detection in uplink MIMO systems due to the large increase in the system dimensions [5]. The maximum likelihood (ML) is the optimal detection approach on data detection, but its computational complexity grows exponentially with the number of user equipment (UE) and the modulation order [6,7]. Some suboptimal detection alternatives are proposed to reduce the computational complexity while obtaining a good bit error rate (BER) performance. For example, the linear minimum mean squared error (LMMSE) algorithm is one of the widely-used suboptimal detection algorithms with near-optimal BER performance and reduced computational complexity [8]. However, the LMMSE algorithm still involves the computation of the Gram matrix, as well as matrix inversion, where their corresponding computational complexity is $O\left(N_{r} N_{t}^{2}\right)$ and $O\left(N_{t}^{3}\right)$, respectively, with $N_{t}$ denoting the number of single-antenna UE and $N_{r}$ denoting the number of antennas at the BS. 
It is worth noting that some approaches for approximating the matrix inversion have been proposed to reduce the computational complexity [9-19], among which, for example, the Neumann series (NS) approximation is used to approximate the matrix inversion by a series of truncated NS expansions $[9,10]$. However, only marginal reduction in the computational complexity can be reached with the increased terms of the NS expansion. Hence, various classical iterative algorithms have been provided to approximate the inverse matrix in LMMSE detection to achieve low computational complexity, which include the Richardson (RI) algorithm [11], the Jacobi algorithm [12-15], the successive over relaxation (SOR) algorithm [16], the symmetric successive over relaxation (SSOR) algorithm [17], and the Gauss-Seidel (GS) algorithm [18,19]. The computational complexity of the matrix inverse is reduced by these approximation-based detection algorithms from $O\left(N_{t}^{3}\right)$ to $O\left(N_{t}^{2}\right)$. It is noted that these algorithms involving the inverse-matrix approximation in LMMSE detection (also known as the approximated LMMSE) achieve a near-LMMSE performance, but with lower computational complexity.

It is well known that the approximated LMMSE detection algorithms achieve a substantial performance loss when $N_{r}>N_{t}$ in multiuser massive MIMO systems. Various algorithms have been proposed to obtain a better BER performance than that of LMMSE detection in multiuser massive MIMO systems, which include the non-convex and the convex optimization algorithms [20-22].

For example, the alternating minimization (AltMin) algorithm is one of the non-convex optimization algorithms, which is applied to the data detection in a multiuser massive MIMO system [20]. Specifically, the AltMin algorithm converts the ML detection problem into a sum of convex functions by decomposing the received vector into multiple sub-vectors. Hence, the non-convex problem is transformed into the convex problem in the AltMin algorithm. The AltMin algorithm shows better BER performance than that of the LMMSE detector in overloaded network scenarios with relatively low computational complexity. However, it shows near-LMMSE performance with even higher computational complexity when the ratio of the number of BS antennas to the number of single-antenna users is larger.

Similarly, some convex optimization algorithms are used to solve the non-convex optimization problems, which include, for example, the alternating direction method of multipliers (ADMM) detection algorithm [21,22]. In the multiuser massive MIMO system, ADMM demonstrates better BER performance than the LMMSE detection algorithm with the relatively low computational complexity of the iterative procedure. However, the computational complexity of the preprocessing in the ADMM algorithm includes the calculation complexity of the Gram matrix and LDLdecomposition [23,24], which results in very high computational complexity for massive MIMO systems.

To make a tradeoff between the performance and the computational complexity with different antenna configurations, the iterative discrete estimation (IDE) algorithm is integrated into the ADMM algorithm [25], which presents low computational complexity due to the avoidance of the calculation of the Gram matrix and LDL decomposition. Motivated by the aforementioned algorithms, we propose a modified IDE (MIDE) algorithm to present a better BER performance and lower computational complexity than the ADMM algorithms. To summarize, the main contributions of this work are listed as follows.

- A novel iterative data detection algorithm for uplink multiuser massive MIMO systems is designed by exploiting the IDE-based algorithmic framework. The proposed MIDE algorithm refactors the detection algorithm as a series of simpler subproblems with closed-form solutions.

- A heuristic damping factor is defined based on the Euclidean distance instead of a fixed factor. Compared with the fixed damping factor, this self-updated damping factor contributes to a faster convergence in the proposed MIDE algorithm.

- The computational complexity analysis indicates that the proposed algorithm has a lower computational complexity than the traditional approximated detection approximation algorithms (LMMSE, AltMin, and ADMM), under the same BER performance. Specifically, the complexity of the novel MIDE detection algorithm is only $O\left(N_{t}^{2}\right)+O\left(N_{r} N_{t}\right)$. 
- Simulation results reveal that for the typical independent and identically distributed (i.i.d.) frequency flat Rayleigh fading channel in massive MIMO systems, the proposed MIDE detection algorithm performs better than the ADMM and AltMin-based detection algorithms and the LMMSE detection algorithm in terms of BER performance with various system configurations.

The rest of the paper is organized as follows. In Section 2, we briefly introduce the system model. Section 3 specifies the proposed low-complexity signal detection based on the IDE algorithm and performs the computational complexity analysis of the algorithms. In Section 4, the numerical simulation results of the BER performance are presented. Finally, Section 5 concludes the paper.

Notation: Throughout the paper, the lowercase and uppercase boldface type is used for vectors (e.g., a) and matrices (e.g., A). The superscripts $(\cdot)^{-1}$ and $(\cdot)^{H}$ denote the matrix inversion and the conjugate transpose, respectively. The $L_{2}$ norms of the vectors are represented by $\|\cdot\|_{2} \cdot \Re$ and $\Im$ denote the real part and the imaginary part of the complex-valued signal, respectively.

The typical uplink massive MIMO system is considered in this work, as shown in Figure 1, where there are $N_{t}$ single-transmitting antenna UE devices and $N_{r}$ receiving antennas at the BS [26]. In general, $N_{r}$ is larger than $N_{t}$ for an uplink massive MIMO communication system [27].

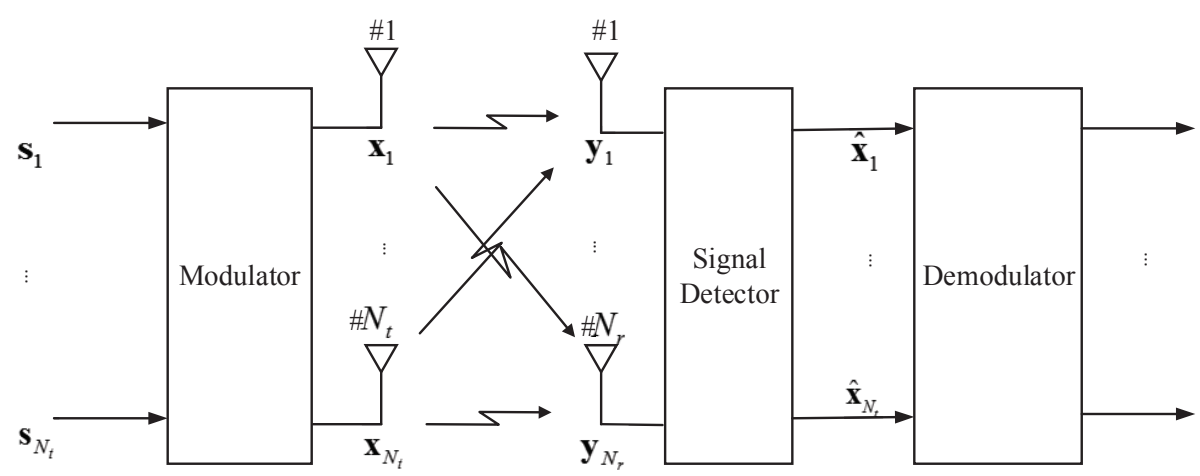

Figure 1. MIMO system signal detection structure diagram.

\section{System Model}

At the transmitter side, the source information $\mathbf{s}=\left[\mathbf{s}_{1}, \cdots, \mathbf{s}_{i}, \cdots \mathbf{s}_{N_{t}}\right]^{T}$ where each symbol $s$ is mapped to constellation symbols by taking symbols from a set of the constellation alphabet $\Omega$. The transmitted signal $\mathbf{x}=\left[\mathbf{x}_{1}, \cdots, \mathbf{x}_{i}, \cdots \mathbf{x}_{N_{t}}\right]^{T}$ is constructed by the modulated symbol s, where $\mathbf{x}_{i}$ denotes a signal transmitted by the $i^{\text {th }}$ UE device. The vector $\mathbf{y}=\left[\mathbf{y}_{1}, \cdots, \mathbf{y}_{i}, \cdots \mathbf{y}_{N_{r}}\right]^{T}$ represents the receiving signal at the $\mathrm{BS}$, where $\mathbf{y}_{i}$ denotes a signal received by the $i^{\text {th }}$ receive antenna, and:

$$
\mathbf{y}=\mathbf{H x}+\mathbf{w}
$$

where $\mathbf{w}$ is the $N_{r}$-by-one additive white Gaussian noise (AWGN) vector following $C N\left(0, \sigma^{2}\right)$ with $\sigma^{2}$ representing the average power of the noise. In Equation (1), the matrix $\mathbf{H}$ denotes the $N_{r}$-by- $N_{t}$ flat fading channel gain, and $\mathbf{H}$ can be expressed as:

$$
\mathbf{H}=\left[\begin{array}{cccc}
h_{11} & h_{12} & \cdots & h_{1 N_{t}} \\
h_{21} & h_{22} & \cdots & h_{2 N_{t}} \\
\vdots & \vdots & \ddots & \vdots \\
h_{N_{r} 1} & h_{N_{r} 2} & \cdots & h_{N_{r} N_{t}}
\end{array}\right]
$$

where the element $h_{i, j}, i=\left\{1,2, \cdots, N_{r}\right\}, j=\left\{1,2, \cdots, N_{t}\right\}$ denotes the channel impulse response between the receiving antenna $j$ and the user $i$. It is noted that $h_{i j}$ follows an i.i.d. Gaussian distribution with zero mean and unit variance. In addition, the channel matrix $\mathbf{H}$ is assumed to be known perfectly at the BS $[28,29]$. 


\section{Proposed MIDE Algorithm}

We first propose a low-complexity signal detection algorithm, which converts the ML problem into the constrained non-convex problem and utilizes the IDE-based algorithm to solve the problem iteratively. Then, a self-updating method is proposed to update the damping factor based on the Euclidean distance, which accelerates the convergence in the proposed MIDE algorithm. Finally, the computational complexity analysis of the proposed algorithm is provided with the comparison to the conventional algorithms.

\subsection{Problem Formulation and IDE-Based Algorithm}

Detecting the transmitted symbol vector $\mathbf{x}$ at the BS can be done by minimizing the squared Euclidean distance between the received signal vector $\mathbf{y}$ and the hypothesized received signal $\mathbf{H x}$ with the vector $\mathbf{x}$ constrained to the modulation constellation $\Omega^{N_{t}}$, which can be represented as:

$$
\hat{\mathbf{x}}=\underset{x \in \Omega^{N_{t}}}{\arg \min }\|\mathbf{y}-\mathbf{H x}\|_{2}^{2}
$$

It is noted that in Equation (3), the finite-alphabet constraint $x \in \Omega^{N_{t}}$ can be converted into the indicator function $\mathbf{I}_{\Omega}(\hat{\mathbf{x}})$, which is given by:

$$
I_{\Omega}(\hat{\mathbf{x}})=\left\{\begin{array}{c}
0, \text { if } \hat{\mathbf{x}} \in \Omega^{N_{t}} \\
\infty, \text { otherwise }
\end{array}\right.
$$

By combining Equation (3) and (4), the signal detection problem can be converted into the constrained optimization problem, which is given by:

$$
\begin{gathered}
\underset{\mathbf{z}, \hat{\mathbf{x}}}{\operatorname{minimize}}\|\mathbf{y}-\mathbf{H z}\|_{2}^{2}+\mathbf{I}_{\Omega}(\hat{\mathbf{x}}) \\
\text { s.t. } \mathbf{z}-\hat{\mathbf{x}}=\mathbf{0}
\end{gathered}
$$

where $\mathbf{z}$ is the least-squares solution of the least-squares and $\hat{\mathbf{x}}$ is the estimated transmitted symbol. Since the optimization problem is defined over complex-valued variables, the Lagrangian objective function for the optimization problem in Equation (5) can be remodeled as:

$$
L_{\gamma}(\mathbf{z}, \hat{\mathbf{x}}, \mathbf{u})=\|\mathbf{y}-\mathbf{H z}\|_{2}^{2}+\mathbf{I}_{\Omega}(\hat{\mathbf{x}})+\gamma\|\mathbf{z}-\hat{\mathbf{x}}\|+\mathbf{u}^{H}(\mathbf{z}-\hat{\mathbf{x}})
$$

where $\gamma>0$ is the penalty parameter and $\mathbf{u}$ is the dual vector. In order to solve this problem efficiently, we decompose it into three sub-problems. First, we solve $\mathbf{z}$ while holding $\hat{\mathbf{x}}$ and $\mathbf{u}$ fixed; then, we solve $\hat{\mathbf{x}}$ while holding $\mathbf{z}$ and $\mathbf{u}$ fixed; finally, we solve $\mathbf{u}$ while holding $\hat{\mathbf{x}}$ and $\mathbf{z}$ fixed. Specifically, the following procedure is repeated with iterations.

$$
\begin{aligned}
& \mathbf{z}^{k}=\underset{\mathbf{z}}{\arg \min } L_{\gamma}\left(\mathbf{z}, \hat{\mathbf{x}}^{k-1}, \mathbf{u}^{k-1}\right) \\
& \hat{\mathbf{x}}^{k}=\underset{\hat{\mathbf{x}}}{\arg \min } L_{\gamma}\left(\mathbf{z}^{k}, \hat{\mathbf{x}}^{k}, \mathbf{u}^{k-1}\right) \\
& \mathbf{u}^{k}=\mathbf{u}^{k-1}+\gamma\left(\mathbf{z}^{k}-\hat{\mathbf{x}}^{k}\right)
\end{aligned}
$$

Note that the $\mathbf{z}$-minimization procedure is convex, but the $\hat{\mathbf{x}}$-update is projected onto a convex set $\Omega^{N_{t}}$. To make the iterative procedure converge, the IDE-based algorithm is applied to remove the dual vector $\mathbf{u}$ at each iterative and turn the $\mathbf{z}$-update and $\hat{\mathbf{x}}$-update to reach a consensus. After manipulation, the $\hat{\mathbf{x}}$-update involves solving a linearly-constrained minimum Euclidean-norm problem, and the z-update in IDE is given by: 


$$
\mathbf{z}^{k}=\hat{\mathbf{x}}^{k-1}+\left[\operatorname{diag}\left(\mathbf{H}^{H} \mathbf{H}\right)\right]^{-1} \mathbf{H}^{H}\left(\mathbf{y}-\mathbf{H} \hat{\mathbf{x}}^{k-1}\right)
$$

Hence, the $\hat{\mathbf{x}}$-update step can be represented as:

$$
\hat{\mathbf{x}}^{k+1}=\Pi_{\Omega}\left(\mathbf{z}^{k+1}\right)
$$

where $\Pi_{\Omega}(\cdot)$ is the projection onto $\Omega^{N_{t}}$, i.e., the elements of $\mathbf{z}^{k+1}$ can be implemented through simple rounding of each component to the closest element in $\Omega$.

Additionally, to make the iteration converge, a fixed damping factor $\alpha$ is employed in the IDE-based algorithm to update the iterative solution $\hat{\mathbf{x}}$, i.e.,

$$
\hat{\mathbf{x}}_{d}^{k+1}=\left(1-\alpha^{k}\right) \hat{\mathbf{x}}_{d}^{k}+\alpha^{k} \hat{\mathbf{x}}^{k+1}
$$

where $\hat{\mathbf{x}}_{d}^{k+1}$ denotes the solution after updating. By applying $\hat{\mathbf{x}}_{d}$ to the $\mathbf{z}$-update, the IDE-based algorithm for detection can be expressed as:

$$
\begin{aligned}
& \mathbf{z}^{k}=\hat{\mathbf{x}}_{d}^{k-1}+\left[\operatorname{diag}\left(\mathbf{H}^{H} \mathbf{H}\right)\right]^{-1} \mathbf{H}^{H}\left(\mathbf{y}-\mathbf{H} \hat{\mathbf{x}}_{d}^{k-1}\right) \\
& \hat{\mathbf{x}}^{k}=\Pi_{\Omega}\left(\mathbf{z}^{k}\right) \\
& \hat{\mathbf{x}}_{d}^{k}=\left(1-\alpha^{k}\right) \hat{\mathbf{x}}_{d}^{k-1}+\alpha^{k} \hat{\mathbf{x}}^{k}
\end{aligned}
$$

\subsection{Modified IDE-Based Detection Algorithm with Self-Update Damping}

The performance of the IDE-based detection algorithm is influenced by the choice of the damping factor. In early studies, this parameter was fixed, e.g., 0.05 in [25]. However, a fixed damping factor is not applicable for all cases, and an optimal damping factor is not easy to obtain. We propose an MIDE herein to decide a proper damping factor $\alpha$, by which the $\hat{\mathbf{x}}_{d}$-update step is analyzed as follows.

$$
\begin{aligned}
\hat{\mathbf{x}}_{d}^{1} & =\left(1-\alpha^{0}\right) \hat{\mathbf{x}}_{d}^{0}+\alpha^{0} \hat{\mathbf{x}}^{1}, \\
\hat{\mathbf{x}}_{d}^{2} & =\left(1-\alpha^{1}\right) \hat{\mathbf{x}}_{d}^{1}+\alpha^{1} \hat{\mathbf{x}}^{2} \\
& =\left(1-\alpha^{1}\right)\left(\left(1-\alpha^{0}\right) \hat{\mathbf{x}}_{d}^{0}+\alpha^{0} \hat{\mathbf{x}}^{1}\right)+\alpha^{1} \hat{\mathbf{x}}^{2} \\
& =\left(1-\alpha^{1}\right)\left(1-\alpha^{0}\right) \hat{\mathbf{x}}_{d}^{0}+\left(1-\alpha^{1}\right) \alpha^{0} \hat{\mathbf{x}}^{1}+\alpha^{1} \hat{\mathbf{x}}^{2} \\
& \vdots \\
\hat{\mathbf{x}}_{d}^{k+1} & =\left(1-\alpha^{k}\right) \hat{\mathbf{x}}_{d}^{k}+\alpha^{k} \hat{\mathbf{x}}^{k+1} \\
& =\left(1-\alpha^{k-1}\right) \cdots\left(1-\alpha^{0}\right) \hat{\mathbf{x}}_{d}^{0}+\left(1-\alpha^{k-1}\right) \cdots\left(1-\alpha^{1}\right) \alpha^{0} \hat{\mathbf{x}}^{1}+\cdots+\alpha^{k-1} \hat{\mathbf{x}}^{k+1}
\end{aligned}
$$

Since there is no prior information of the final result $\hat{\mathbf{x}}_{d}$, the initial value of $\hat{\mathbf{x}}_{d}^{0}$ can be set as a zero vector. Hence, the expression for $\hat{\mathbf{x}}_{d}$ in Equation (16) is composed of the solution of $\hat{\mathbf{x}}$ with different values of the damping factor. Based on the expression in Equation (16), the convergence of the iterations can be measured by the difference between $\hat{\mathbf{x}}_{d}^{k}$ and $\hat{\mathbf{x}}^{k+1}$. Specifically, the Euclidean distance, which is one of the widely-used approaches for measuring the distance between two vectors [30], is defined as:

$$
d^{k}\left(\hat{\mathbf{x}}_{d}^{k}, \hat{\mathbf{x}}^{k+1}\right)=\sqrt{\sum_{i=1}^{N_{t}}\left(\hat{\mathbf{x}}_{d}^{k}(i, 1)-\hat{\mathbf{x}}^{k+1}(i, 1)\right)^{2}}
$$


We can notice from Equation (17) that the smaller the distance d, the closer the two vectors, implying the convergence of the IDE iterations. Based on this discussion, the following heuristic damping factor at the $k^{\text {th }}$ iteration is defined as:

$$
\alpha^{k}=\frac{d^{k}}{d^{k}+q}
$$

where $q$ is a positive constant. Obviously, a higher value of $d$ leads to a higher $\alpha$, and vice versa. In other words, when $d^{k} \rightarrow 0, \alpha^{k} \rightarrow 0$, and when $d^{k} \rightarrow 1, \alpha^{k} \rightarrow 1$. We utilize the first iteration result $d^{1}$ to obtain the value of $p$. According to Equation (16), $d^{1}$ can be calculated as:

$$
\begin{aligned}
d^{1} & =\sqrt{\sum_{i=1}^{N_{t}}\left(\hat{\mathbf{x}}_{d}^{0}(i, 1)-\hat{\mathbf{x}}^{1}(i, 1)\right)^{2}} \\
& =\sqrt{\sum_{i=1}^{N_{t}}\left(\hat{\mathbf{x}}^{1}(i, 1)\right)^{2}} \\
& =\sqrt{\sum_{i=1}^{N_{t}}\left(\Re\left(\hat{\mathbf{x}}^{1}(i, 1)\right)\right)^{2}+\left(\Im\left(\hat{\mathbf{x}}^{1}(i, 1)\right)\right)^{2}}
\end{aligned}
$$

It is noticed that the value of $d^{1}$ is varying and decided based on several factors such as the modulation method, the noise in the system, etc. For the ease of calculation, the expectation of $d^{1}$ is computed instead of the direct calculation of $d^{1}$. Since the real part and the imaginary part of the vector $\hat{\mathbf{x}}^{1}$ have the same uniform distribution, the expectation for $d^{1}$ is obtained as:

$$
E\left(d^{1}\right)=2 E\left(\sqrt{\sum_{i=1}^{N_{t}}\left(\Re\left(\hat{\mathbf{x}}^{1}(i, 1)\right)\right)^{2}}\right)
$$

The expectation of $d^{1}$ is based on the constellation points of the modulation scheme. With 16-QAM, for example, $\Re\left(\hat{\mathbf{x}}^{1}(i, 1)\right)$ can be $\{-3,-1,+1,+3\}$, and the probability $p$ of each possible value of the point is the same without the prior information, i.e., $p=0.25$ in this case. Hence, Equation (19) can be rewritten as:

$$
E\left(d^{1}\right)=2 E\left(\sqrt{\sum_{i=1}^{N_{t}}\left(\Re\left(\hat{\mathbf{x}}^{1}(i, 1)\right)\right)^{2}}\right)=2 N_{t} \sqrt{\frac{\sum c a n d^{2}}{\sqrt{M}}}
$$

where cand represents the candidate value set of $\Re\left(\hat{\mathbf{x}}^{1}(i, 1)\right)$ and $M$ represents the modulation cardinality. For the ease of description, we still take the 16-QAM as an example. Then, cand is denoted as the candidate values $\{-3,-1,+1,+3\}$ and $\sum c a n d^{2}=(-3)^{2}+(-1)^{2}+1^{2}+3^{2} . M$ represents the modulation cardinality. Based on experience, the value of $\alpha^{1}=0.8$ is set as 0.8 . Then, substituting Equation (22) into Equation (18) yields the estimation of $q$ as:

$$
q=\frac{E\left(d^{1}\right)}{4}=\frac{N_{t}}{2} \sqrt{\frac{\sum c a n d^{2}}{\sqrt{M}}}
$$

The simulation results in Section 4 show that the proposed MIDE algorithm can improve the BER performance significantly compared to the IDE-based algorithm, which employs the fixed damping factor. The procedure of the proposed MIDE detection algorithm is illustrated in Algorithm 1. 


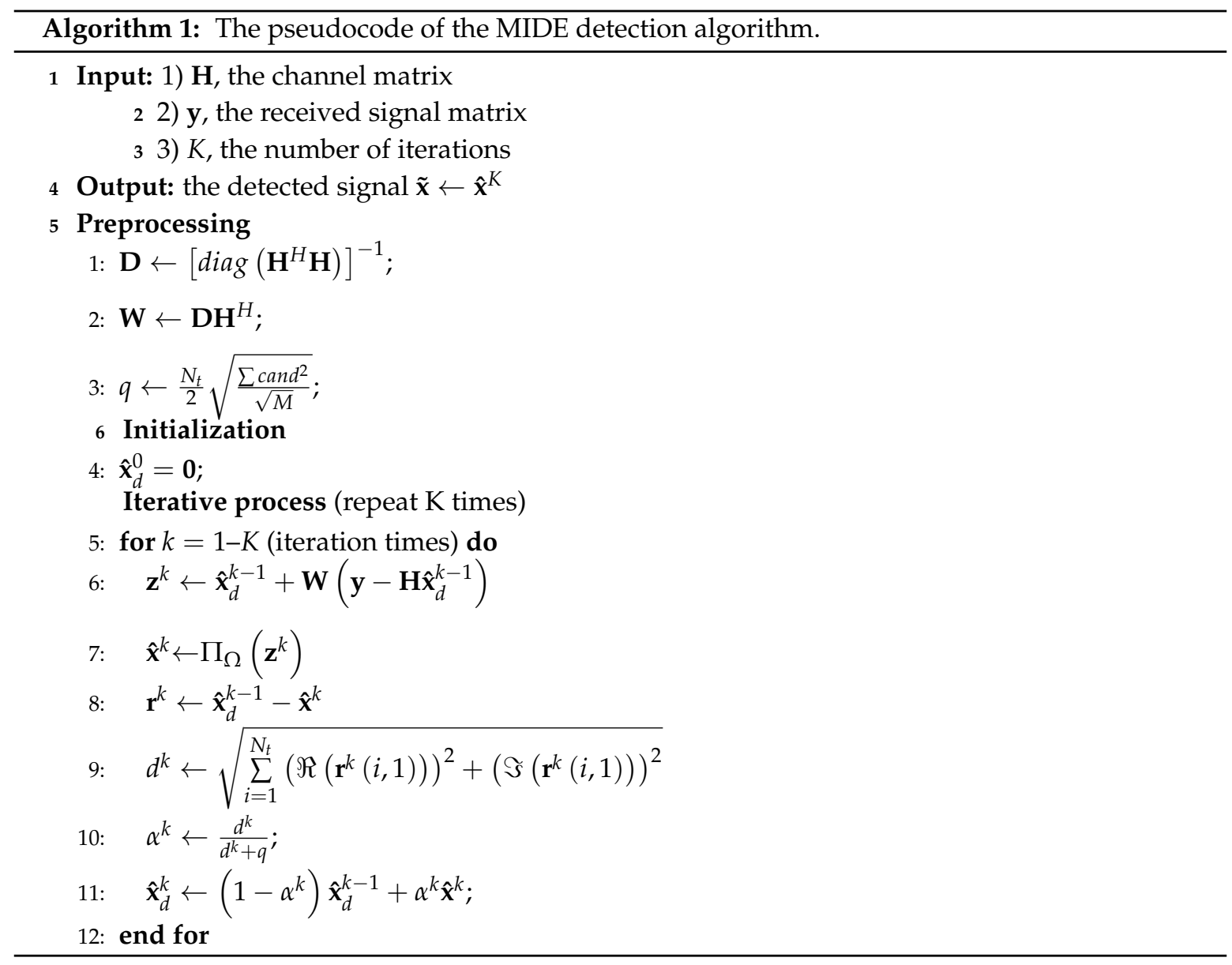

\subsection{Analysis of the Complexity of the Algorithm}

In this subsection, we analyze the computational complexity of the proposed MIDE algorithm, which is dominated by the multiplications operations. Hence, we compute the number of complex-valued multiplications as the measurement of the computational complexity of the algorithm [31].

It can be found in Algorithm 1 that the computational complexity is composed of three parts including (1) preprocess, (2) the $\mathbf{x}$-update procedure, and (3) the $\mathbf{x}_{d}$-update procedure.

(1) Preprocess: The first part comes from the related computation before the iterative process. The main factors affecting the computational complexity of the preprocess are the computation of $\mathbf{D}$ and the multiplication of the $N_{t} \times N_{t}$ diagonal matrix and the $N_{t} \times N_{r}$ matrix. Let $\mathbf{h}_{i}$ be the $i^{\text {th }}$ column of the complex-valued channel matrix $\mathbf{H}$. Then, the diagonal calculation can be presented as:

$$
\mathbf{D}_{i j}=\left\{\begin{array}{c}
\frac{1}{\mathbf{h}_{i}^{H} \mathbf{h}_{j}}, \quad i=j \\
0, \quad i \neq j
\end{array}\right.
$$

Therefore, the complexity of the preprocessing is counted as $N_{t}^{2}+N_{r} N_{t}$.

(2) $\mathbf{x}$-update procedure: The second part comes from the $\mathrm{x}$-update procedure, which involves the computation of two multiplications of the $N_{t} \times N_{t}$ matrix and the $N_{t} \times 1$ vector. Thus, the complexity is counted as $2 N_{r} N_{t}$.

(3) $\mathbf{x}_{d}$-update procedure: The third part originates from updating the value of $\mathbf{x}_{d}$. As can be seen in the expression of $\mathbf{x}_{d^{\prime}}^{k}$, the computation of this part includes the update of the Euclidean distance $d^{k}$ 
and two scalar multiplications with $N_{t} \times 1$ vectors. Then, the complexity in this part is counted as $3 N_{t}$.

Therefore, the overall required number of complex multiplications by the MIDE algorithm is $N_{t} N_{r}+N_{t}^{2}+K\left(2 N_{r} N_{t}+3 N_{t}\right)$. For comparison, the similar calculations of the complexity of the typical detection algorithms (e.g., LMMSE with full matrix inversion, AltMin, ADMM) are given, as well as the one of the proposed MIDE in Table 1.

Table 1. Complexity comparison in terms of complex multiplication operations. LMMSE, linear minimum mean squared error; AltMin, alternating minimization; ADMM, alternating direction method of multipliers; MIDE, modified iterative discrete estimation.

\begin{tabular}{cc}
\hline Algorithm & Complexity \\
\hline LMMSE [8] & $\frac{5}{6} N_{t}^{3}+\frac{3}{4} N_{t}^{2}+\frac{4}{3} N_{t}+N_{r} N_{t}^{2}+N_{r} N_{t}$ \\
AltMin [18] & $(4 K+2) N_{r} N_{t}$ \\
ADMM [20] & $\frac{1}{2} N_{t}^{3}+N_{t}^{2}+N_{r} N_{t}^{2}+N_{r} N_{t}+K\left(N_{t}^{2}+N_{r} N_{t}\right)$ \\
MIDE & $N_{t} N_{r}+N_{t}^{2}+K\left(2 N_{r} N_{t}+3 N_{t}\right)$ \\
\hline
\end{tabular}

Note that all these algorithms utilize the approximation approaches to solve the ML problem. It is obvious that the proposed MIDE algorithm and AltMin algorithm have lower computational complexity at each iteration among the compared iterative approaches. The numerical analysis will be provided for further analysis of the computational complexity, which depends on the number of iterations $K$ and is presented in Section 4.

\section{Simulation Results}

\subsection{BER Performance Evaluation}

The BER performance of the proposed algorithm was evaluated and compared with the ones of other detection algorithms by numerical simulations. The simulation parameters are listed in Table 2 . Several typical detection algorithms were selected for comparison, which were introduced in Section 1 and listed as LMMSE, AltMin, and ADMM.

We first considered the number of iterations for the antenna configurations of $N_{t} \times N_{r}=16 \times 128$, $32 \times 128$ and $64 \times 128$. Figure 2 illustrates the BER performance of the proposed MIDE detection algorithm against the number of iterations, where the SNR was set as $3 \mathrm{~dB}$. It is observed that with the increase of $N_{r} / N_{t}$, the convergence number of the iterations required by the proposed MIDE was almost the same and a very small one, e.g., 10 in all simulations. This demonstrated a reliable BER performance and a fast detection convergence in our proposed MIDE algorithm. Moreover, with the increase of the transmitting antennas, the diversity gain of the MIMO system decreased, leading to a degradation of the BER performance of the proposed algorithm and the compared MIMO detectors.

Table 2. Simulation parameters.

\begin{tabular}{cc}
\hline Channel Model & Uncorrelated Rayleigh Flat Fading \\
\hline Modulation scheme & $16-\mathrm{QAM}$ \\
Number of transmitting antennas $\left(N_{t}\right)$ & $16,32,64$ \\
Number of receiving antennas $\left(N_{r}\right)$ & 128 \\
\hline
\end{tabular}




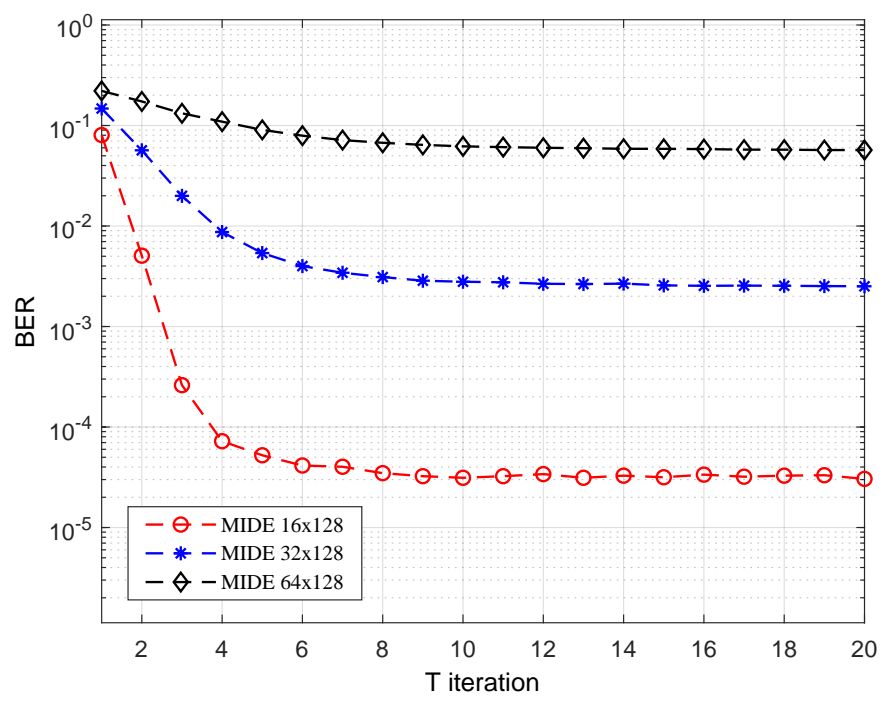

Figure 2. BER performance versus proposed iterations with $\mathrm{SNR}=3 \mathrm{~dB}$.

Figure 3 depicts the BER performance comparison of the IDE-based detection algorithm with the fixed damping factor $\alpha=0.05$ and the proposed MIDE detection with the self-updated damping factor. It is clear that the proposed MIDE-based detection with the self-updated damping factor showed better BER performance than the conventional IDE-based detection algorithm with all antenna configurations.

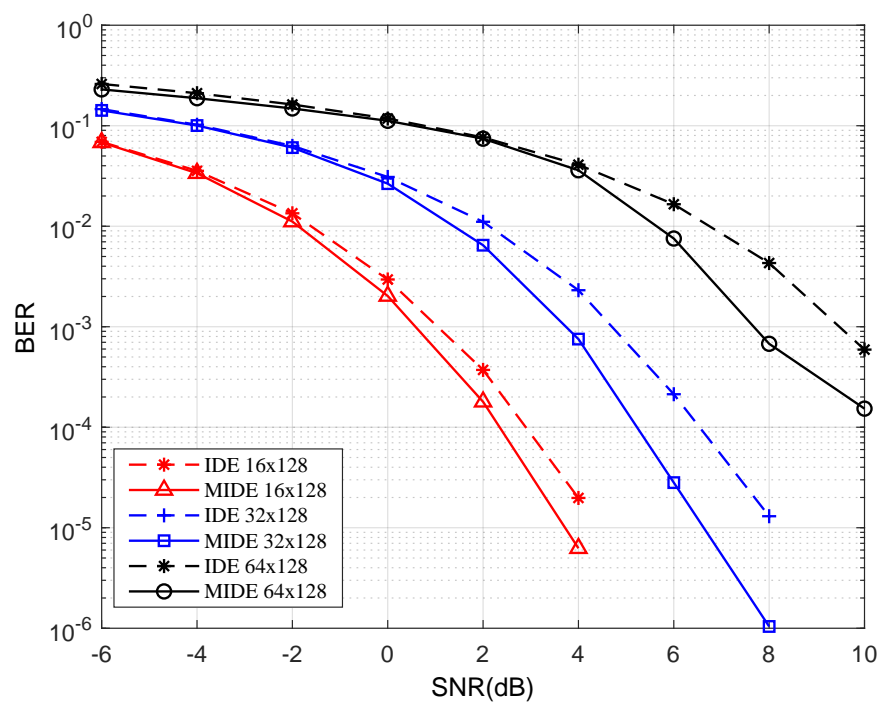

Figure 3. BER performance of the conventional damping factor and the proposed damping factor.

Moreover, Figure 4 compares the BER performances of the proposed MIDE algorithm and the conventional ADMM algorithm. From the figure, it is clear that the BER performance of both algorithms degraded when the number of users increased. However, it is observed that the proposed MIDE-based detection performed better than the conventional ADMM detection in terms of BER performance with all antenna configurations. Furthermore, we can observe from Figure 4 that when the target of BER was set as $10^{-3}$, the SNR required by the proposed algorithm was at least $0.5 \mathrm{~dB}$ less than the one of the conventional ADMM algorithm. 


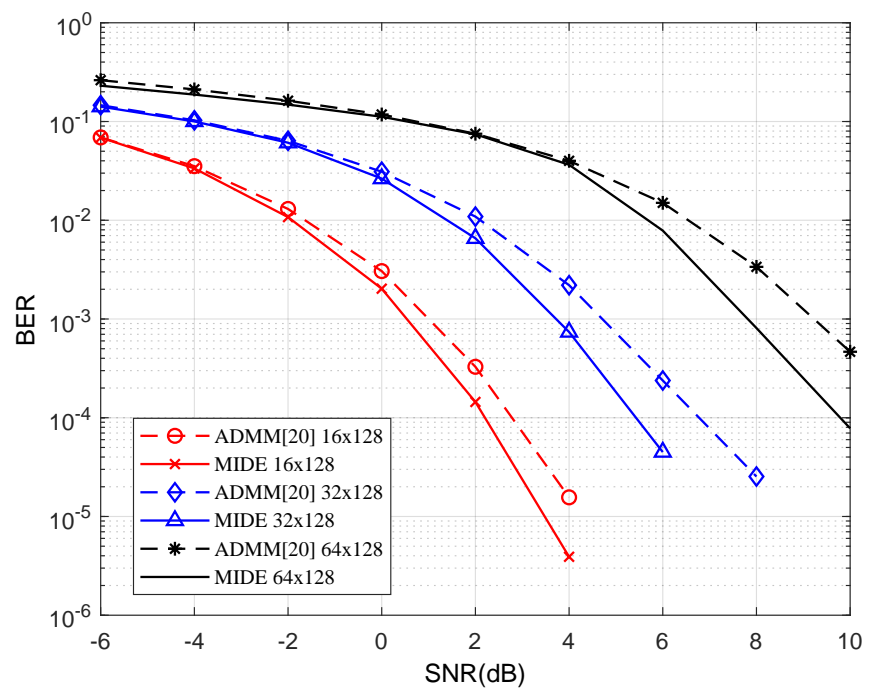

Figure 4. BER performance comparison of the conventional ADMM and the proposed algorithm.

Finally, we show the BER performance comparison of the conventional AltMin algorithm, the LMMSE algorithm, and the proposed MIDE algorithm in Figure 5.

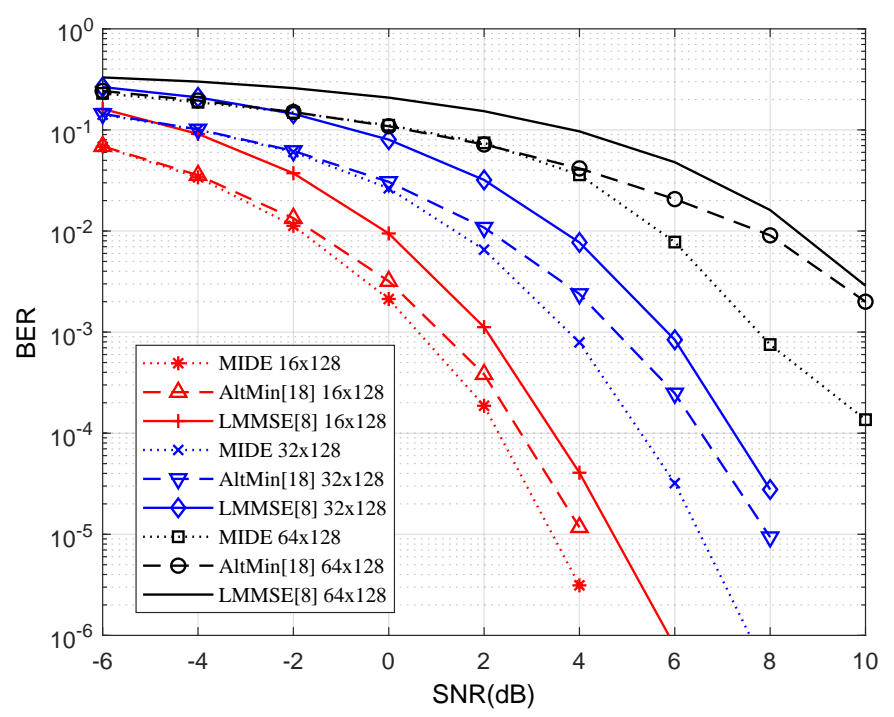

Figure 5. BER performance comparison of the AltMin algorithm, the LMMSE algorithm, and the proposed algorithm.

It is clear that with any ratio of $N_{r} / N_{t}$, the proposed MIDE algorithm performed better than all the compared algorithms in terms of BER performance.

\subsection{Computational Complexity Comparison}

The computational complexity of ADMM, AltMin, and the proposed MIDE algorithms depends on the number of iterations $K$. The compared algorithms had a different number of iterations to reach convergence with different antenna configurations. We fixed the number of receiving antennas to $N_{r}=128$, and the number of transmitting antennas $N_{t}$ was increased from 16 to 84 . Further, we set $K=5,14$, and 10 for the conventional ADMM algorithm, the AltMin algorithm [20], and the proposed MIDE algorithm, respectively, based on the convergence simulations. Figure 6 illustrates the total 
number of multiplications vs. the number of transmitting antennas, which was based on the analysis of the computational complexity of the algorithms in Section 3.3.

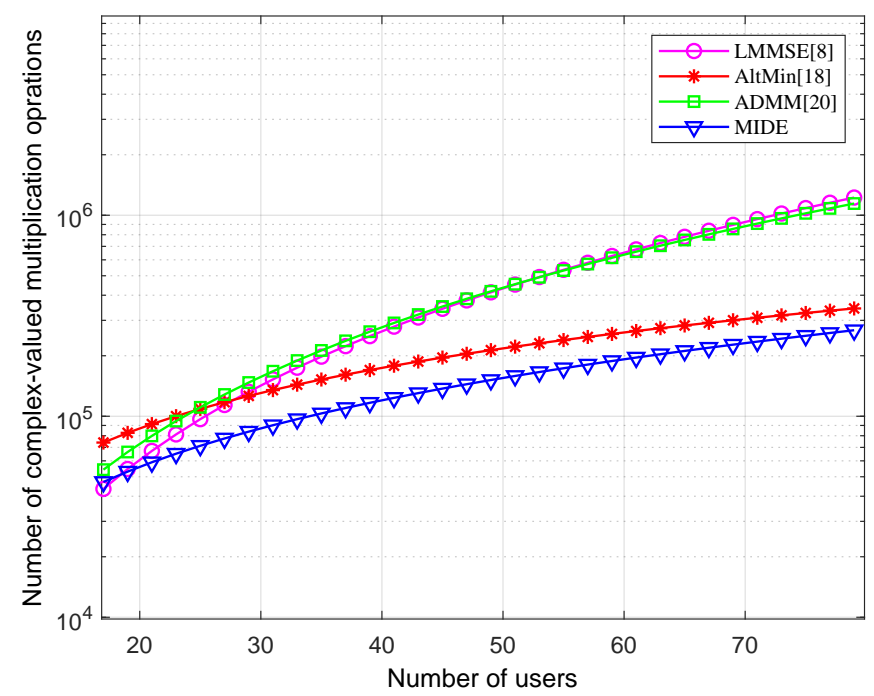

Figure 6. Computational complexity comparison against the number of users.

From Figure 6, we can see that the computational complexity increased with the number of users in all compared algorithms. However, the proposed MIDE algorithm achieved the lowest computational complexity among all compared algorithms under various antenna configurations. Specifically, the MIDE algorithm showed a relatively lower computational complexity than the AltMin algorithm, which was proven to have a low complexity detection for uplink massive systems in [20]. In addition, from Figure 6, the proposed MIDE algorithm achieved much lower computational complexity than the LMMSE and ADMM detection algorithms when the dimension of the MIMO system became larger. As a consequence, the proposed MIDE detection is much more applicable for massive MIMO systems with its low computational complexity.

\section{Conclusions}

In this paper, we proposed a low-complexity, IDE-based detection algorithm in uplink massive MIMO systems. The proposed MIDE algorithm avoided the calculation of the Gram matrix, the matrix inversion, and LDL decomposition to reach a low computational complexity. In addition, a self-updating damping method was provided with the damping factor estimated and updated at each iteration based on the Euclidean distance between the latest two detection solutions, which accelerated the convergence of the IDE-based detection algorithms. Simulation results showed that the proposed MIDE algorithm performed better than the conventional LMMSE, AltMin, and ADMM detection algorithms in terms of the BER performance and the computational complexity.

Author Contributions: H.F. and X.Z. contributed the idea generation; S.X. and X.Z. conducted the study design and paper writing; Z.L. helped the analysis of the simulation results.

Funding: This work is supported in part by the National Natural Science Foundation of China (No. 61571108, No. 61701197, and No. 61801193), the open research fund of National Mobile Communications Research Laboratory of millimeter wave, Southeast University (No. 2018D15), the open research fund of the National Key Laboratory of millimeter wave, Southeast University (No.K201918), the Open Foundation of Key Laboratory of Wireless Communication, Nanjing University of Posts and Telecommunication (No. 2017WICOM01), and the Wuxi Science and Technology Development Fund (No. H20191001).

Conflicts of Interest: The authors declare no conflict of interest. 


\section{References}

1. Xiang, W.; Zheng, K.; Shen, X.S. 5G Mobile Communications; Springer International Publishing: Basel, Switzerland, 2017.

2. Lu, L.; Li, G.Y.A.; Swindlehurst, L.; Ashikhmin, A.; Zhang, R. An overview of massive MIMO: Benefits and challenges. IEEE J. Sel. Topics Signal Process. 2014, 8, 742-758. [CrossRef]

3. Wu, M.; Yin, B.; Wang, G.; Dick, C.; Cavallaro, J.R.; Studer, C. Large-scale MIMO detection for 3GPP LTE: algorithms and FPGA implementations. IEEE J. Sel. Topics Signal Process. 2014, 8, 916-929. [CrossRef]

4. Ngo, H.; Larsson, E.; Marzetta, T. Energy and spectral efficiency of very large multiuser MIMO systems. IEEE Trans. Commun. 2012, 61, 1436-1449.

5. Rusek, F.; Persson, D.; Lau, B.K.; Larsson, E.G.; Marzetta, T. L.; Edfors, O.; Tufvesson, F. Scaling up MIMO: Opportunities and challenges with very large arrays. IEEE Signal Process. Mag. 2013, 30, 40-60. [CrossRef]

6. Chockalingam, A.; Rajan, B.S. Large MIMO Systems; Cambridge University Press: Cambridge, UK, 2014.

7. Kuchi, K.; Ayyar, A.B. Performance analysis of ML detection in MIMO systems with co-channel interference. IEEE Commun. Lett. 2011, 15, 786-788. [CrossRef]

8. Mandloi, M.; Bhatia, V. Low-complexity near-optimal iterative sequential detection for uplink massive MIMO systems. IEEE Commun. Lett. 2017, 21, 568-571. [CrossRef]

9. Gustafsson, O.; Bertilsson, E.; Klasson, J.; Ingemarsson, C. Approximate Neumann Series or exact matrix inversion for massive MIMO? In Proceedings of the 2017 IEEE 24th Symposium on Computer Arithmetic (ARITH), London, UK, 24-26 July 2017.

10. Fang, L.; Huang, D. Neumann Series Expansion Based LMMSE Channel Estimation for OFDM Systems. IEEE Commun. Lett. 2016, 20, 748-751. [CrossRef]

11. Gao, X.; Dai, L.; Ma, Y.; Wang, Z. Low-complexity near-optimalsignal detection for uplink large-scale MIMO systems. Electron. Lett. 2014, 50, 1326-1328. [CrossRef]

12. Qin, X.; Yan, Z.; He, G. A near-optimal detection scheme based on joint steepest descent and Jacobi method for uplink massive MIMO systems. IEEE Commun. Lett. 2016, 20, 276-279. [CrossRef]

13. Lee, Y. Decision-aided Jacobi iteration for signal detection in massive MIMO systems. Electron. Lett. 2017, 53, 1552-1554. [CrossRef]

14. Kong, B.Y.; Park, I. Low-complexity symbol detection for massive MIMO uplink based on Jacobi method. In Proceedings of the IEEE 27th Annual International Symposium on Personal, Indoor, and Mobile Radio Communications (PIMRC), Valencia, Spain, 1-5 September 2016.

15. Minango, J.; de Almeida, C.; Daniel Altamirano, C. Low-complexity MMSE detector for massive MIMO systems based on Damped Jacobi method. In Proceedings of the 2017 IEEE 28th Annual International Symposium on Personal, Indoor, and Mobile Radio Communications (PIMRC), Montreal, QC, Canada, 1-5 October 2017.

16. Deng, Q.; Guo, L.; Dong, C.; Lin, J.; Meng, D.; Chen, X. High-throughput signal detection based on fast matrix inversion updates for uplink massive multiuser multiple-input multi-output systems. IET Commun. 2017, 11, 2228-2235. [CrossRef]

17. Sun, Y.; Li, Z.; Zhang, C.; Zhang, R.; Yan, F.; Shen, L. Low complexity signal detector based on SSOR iteration for large-scale MIMO systems. In Proceedings of the 9th International Conference on Wireless Communications and Signal Processing (WCSP), Nanjing, China, 1-6 October 2017.

18. Dai, L.; Gao, X.; Su, X.; Han, S.; Chih-Lin, I.; Wang, Z. Low-complexity soft-output signal detection based on Gauss-Seidel method for uplink multiuser large-scale MIMO systems. IEEE Trans. Veh. Technol. 2015, 64, 4839-4845. [CrossRef]

19. Zeng, J.; Lin, J.; Wang, Z. An improved Gauss-Seidel algorithm and its efficient architecture for massive MIMO systems. IEEE Trans. Circuits Syst. II-Express Briefs. 2018, 65, 1194-1198. [CrossRef]

20. Elgabli, A.; Elghariani, A.; Aggarwal, V.; Bell, M.R. A low-complexity detection algorithm for uplink massive MIMO systems based on Alternating Minimization. IEEE Wirel. Commun. Lett. 2019, 8, 917-920. [CrossRef]

21. Souto, N.; Dinis, R. MIMO detection and equalization for single-carrier systems using the Alternating Direction Method of Multipliers. IEEE Signal Process. Lett. 2016, 23 , 1751-1755. [CrossRef]

22. Shahabuddin, S.; Juntti, M.; Studer, C. Admm-based infinity normdetection for large MU-MIMO: Algorithm and vlsi architecture. In Proceedings of the IEEE International Symposium on Circuits and Systems (ISCAS), Baltimore, MD, USA,1-4 May 2017. 
23. Zhang, K.; Zhang, Y. Recursive algorithm for calculating eigenvalues of real symmetric matrix based on LDLT decomposition. Computer Engineering and Applications. 2008, 44, 78-80.

24. Chou, C.; Wu, J. Low-complexity MIMO precoder design with LDL $^{H}$ channel decomposition. IEEE Trans. Veh. Technol. 2011, 60, 2368-2372. [CrossRef]

25. Wang, C.; Wen, C.; Jin, S.; Tsai, S. Finite-Alphabet precoding for massive MU-MIMO with low-resolution DACs. IEEE Trans. Wirel. Commun. 2018, 17, 4706-4720. [CrossRef]

26. Chen, J. A low complexity data detection algorithm for uplink multiuser massive MIMO systems. IEEE J. Sel. Areas Commun. 2017, 35, 1701-1714. [CrossRef]

27. Jin, F.; Liu, Q.; Liu, H.; Wu, P. A low complexity signal detection scheme based on improved Newton iteration for massive MIMO systems. IEEE Commun. Lett. 2019, 23, 748-751. [CrossRef]

28. Wu, Z.; Ge, L.; You, X.; Zhang, C. Efficient near-MMSE detector for large-scale MIMO systems. In Proceedings of the 2017 IEEE International Workshop on Signal Processing Systems (SiPS), Lorient, France, 1-6 October 2017.

29. Wu, C.; Huang, W.; Chung, W. Robust update algorithms for Zero-Forcing detection in uplink large-scale MIMO systems. IEEE Commun. Lett. 2018, 22, 424-427. [CrossRef]

30. Monteiro, F.A.; Wassell, I.J. Euclidean distances in quantized spaces with pre-stored components for MIMO detection. In Proceedings of the European Conference on Wireless Technologies, Munich, Germany, 8-10 October 2007.

31. Hunger, R. Floating Point Operations in Matrix-Vector Calculus; Technical Report; Munich University of Technology: Munich, Germany, 2007.

(C) 2019 by the authors. Licensee MDPI, Basel, Switzerland. This article is an open access article distributed under the terms and conditions of the Creative Commons Attribution (CC BY) license (http:/ / creativecommons.org/licenses/by/4.0/). 